\title{
Thermophysical properties of $\mathrm{U}_{3} \mathrm{Si}_{5}$ to $1773 \mathrm{~K}$
}

\author{
J.T. White ${ }^{\mathrm{a}, *}$, A.T. Nelson ${ }^{\mathrm{a}}$, D.D. Byler ${ }^{\mathrm{a}}$, D.J. Safarik ${ }^{\mathrm{a}}$, J.T. Dunwoody ${ }^{\mathrm{a}}$, \\ K.J. McClellan ${ }^{\mathrm{a}}$ \\ ${ }^{a}$ Material Science and Technology Division, Los Alamos National Laboratory, Los \\ Alamos, NM, United States
}

\begin{abstract}
Possible use of $\mathrm{U}_{3} \mathrm{Si}_{5}$ as a nuclear reactor fuel requires knowledge of its thermophysical properties as a function of temperature. While limited data is available for U-Si compounds containing higher uranium densities, no investigations of $\mathrm{U}_{3} \mathrm{Si}_{5}$ have been presented in the literature to date. High purity $\mathrm{U}_{3} \mathrm{Si}_{5}$ was fabricated to facilitate a set of experiments to determine the coefficient of thermal expansion, heat capacity, thermal diffusivity, and thermal conductivity from room temperature to $1773 \mathrm{~K}$. Each measurement on nearly stoichiometric $\mathrm{U}_{3} \mathrm{Si}_{5}$ showed the existence of a phase transformation at $723 \mathrm{~K}$, which is not consistent with the most recently published phase diagram.
\end{abstract}

Keywords: Uranium silicide, $\mathrm{U}_{3} \mathrm{Si}_{5}$, laser flash analysis, thermal conductivity

${ }^{*}$ Corresponding author

Email address: jtwhite@lanl.gov (J.T. White)

Preprint submitted to Journal of Nuclear Materials

October 7, 2014 


\section{Introduction}

Compounds from the U-Si binary system have received historic interest as nuclear reactor fuels for specialized applications. Most familiar of these are $\mathrm{U}_{3} \mathrm{Si}$ and $\mathrm{U}_{3} \mathrm{Si}_{2}$, which have been used for many years as fuels for research reactors [1]. U-Si compounds with higher silicon contents such as $\mathrm{U}_{3} \mathrm{Si}_{5}$ have been rarely investigated due to a uranium density $\left(7.5 \mathrm{~g}-\mathrm{U} / \mathrm{cm}^{3}\right)$ significantly lower than both $\mathrm{U}_{3} \mathrm{Si}$ (15.0) and $\mathrm{U}_{3} \mathrm{Si}_{2}$ (11.3). The uranium density of $\mathrm{U}_{3} \mathrm{Si}_{5}$ is also appreciably lower than $\mathrm{UO}_{2}(9.7)$, more closely matching that of $\mathrm{U}_{3} \mathrm{O}_{8}$ (7.1). This suggests that use of $\mathrm{U}_{3} \mathrm{Si}_{5}$ as a reactor fuel could only be broadly envisioned if high enrichment was allowed for specialty reactor designs, or as a second fissile phase in a composite fuel where a companion high density fuel would increase the total uranium loading to necessary levels. The primary advantages of $\mathrm{U}_{3} \mathrm{Si}_{5}$ identified to date are the neutronic similarity of a $\mathrm{UN}-\mathrm{U}_{3} \mathrm{Si}_{5}$ composite to reference $\mathrm{UO}_{2}$ [2], which would minimize LWR operational differences from the viewpoint of the reactor operator, and greatly improved oxidation resistance compared to the more commonly cited U-Si fuel candidate, $\mathrm{U}_{3} \mathrm{Si}_{2}$ [3]. Although at this point such a fuel is hypothetical, reactor performance modeling would undoubtedly be an early investment to explore neutronics and burnup as a function of possible reactor designs, fuel loading, and many other parameters.

The importance of temperature in governing many aspects of reactor performance demands knowledge of thermophysical properties from room temperature to anticipated operating conditions and through temperatures brought about by potential transients. Even the more familiar U-Si compounds $\mathrm{U}_{3} \mathrm{Si}$ and $\mathrm{U}_{3} \mathrm{Si}_{2}$ have, until recently [4], received minimal attention with respect to their thermophysical properties at elevated temperatures. This is primarily due to lower temperature conditions of research reactors. To date, only a few studies could be located in the literature which focus on the thermophysical properties of $\mathrm{U}_{3} \mathrm{Si}_{5}$ these consisted of sub-ambient investigations focused on fundamental properties.

This study was initiated to provide thermophysical properties for $\mathrm{U}_{3} \mathrm{Si}_{5}$ that can be used by the modeling and simulation communities to test reactor performance prior to in-pile testing. Performance of $\mathrm{U}_{3} \mathrm{Si}_{5}$ fuel under potentially off-normal conditions can also be elucidated from the high temperature data, useful for calculating accident tolerance in LWR safety design criteria. 


\section{Experimental}

Preparation of homogeneous single phase $\mathrm{U}_{3} \mathrm{Si}_{5}$ in geometries suitable for thermophysical property measurement using differential scanning calorimetry (DSC), dilatometry, and laser flash analysis (LFA) was first necessary for execution of this work. Arc melting was chosen as the most efficient means to synthesize gram quantities of $\mathrm{U}_{3} \mathrm{Si}_{5}$ for subsequent fabrication into samples suitable for property measurement. Efforts were taken to prevent $\mathrm{O}_{2}(\mathrm{~g})$ contamination during the fabrication steps to minimize formation of oxide phases. Finally, microstructural characterization was essential to facilitate discussion of the phase content of the material measured.

\subsection{Sample preparation}

High purity feedstock of depleted uranium plate was used as the starting material. Inductively coupled mass spectrometry was conducted to verify impurity content of the U feedstock. Impurities for the uranium metal were below the detectable limit for all impurities with the exception of $31 \mathrm{ppm}$ carbon. The plate was then wire electro-discharge machined into discs approximately 10 grams each. The silicon used was Alfa Aesar stock with 99.999\% purity in the form of small irregular shapes up to $5 \mathrm{~mm}$ in size. The silicon charge was measured such that stoichiometric $\mathrm{U}_{3} \mathrm{Si}_{5}$ would be achieved. Samples were weighed before and after each arc melting to confirm that minimal mass loss occurred during the arc melting process.

Buttons of $\mathrm{U}_{3} \mathrm{Si}_{5}$ were prepared by arc-melting the uranium and silicon feedstock to produce an approximate mass of $10 \mathrm{~g}$ each. Fabrication was performed in an arc melter (5TA Reed Tri-Arc, Centorr Vacuum Industries, USA) constant current welder connected to a water cooled $2 \%$ thoriated tungsten electrode and a water cooled copper hearth. A gettered argon gas stream fed the arc melter chamber with an oxygen mole fraction $\left(\chi_{\mathrm{O}_{2}}\right)$ less than $10^{-10}$ parts per million (ppm). A molten titanium getter was also utilized inside the arc melter itself to capture any remaining oxygen in the chamber. Following arc melting, $\mathrm{U}_{3} \mathrm{Si}_{5}$ buttons were heat treated at $1570 \mathrm{~K}$ for 48 hours in a $\mathrm{W}$-metal furnace to homogenize the solidified melt. $\mathrm{U}_{3} \mathrm{Si}_{5}$ is reported to form congruently at $2043 \mathrm{~K}$ according the most recent published phase diagram [5].

\subsection{Sintering $U_{3} S_{5}$ powder}

Specimens for thermophysical property measurements were prepared by a powder metallurgy route. Arc-melted $\mathrm{U}_{3} \mathrm{Si}_{5}$ buttons were comminuted 
in a high energy SPEX mill (8000M, SPEX Sample Prep, USA) using a stainless steel jar and milling media. The resulting powder was sieved to -200 mesh within a nitrogen glove box maintained between 1-20 ppm $\mathrm{O}_{2}$ concentration to minimize oxidation of the powder. Sieved powders were then pressed at $300 \mathrm{MPa}$ in a steel die set, which utilized $\mathrm{Zn}$ stearate as a die lubricant. Binders were not used during fabrication of the pellets in order to minimize oxygen contamination. Pellets were sintered using a W metal furnace on Ta foil under flowing Ar gas. The Ar gas stream was gettered through a $\mathrm{Cu}$ charge to minimize the oxygen content in the gas stream, which was monitored throughout the furnace profile. Densities were determined through immersion density measurement according to ASTM B 962-08 [6] using Flourinert FC-43 as the immersion fluid. The balance used to determine masses in this study was calibrated to $0.01 \mathrm{mg}$. Resulting pellets were between $96-98 \%$ of the theoretical density of $\mathrm{U}_{3} \mathrm{Si}_{5}$ taken as $9.06 \mathrm{~g} / \mathrm{cm}^{3}$.

Representative samples for chemical analysis were taken from the comminuted button that was used to fabricate the pellets used in this study. Inductively coupled plasma-optical emission spectroscopy (ICP-OES) was used to determine transition and transuranic elements, while the $\mathrm{WO}_{3}$ combustion catalyst method was utilized to determine $\mathrm{H}, \mathrm{C}$, and $\mathrm{N}$ content. Elemental $\mathrm{O}$ analysis is not possible using this experimental technique, and was therefore not evaluated in this study. ICP-OES required $50 \mathrm{mg}$ of powder to be digested in a solution of nitric, hydrofluoric, and hydrochloric acid. Chemical analysis was by ALS Environmental of Tuscon, Arizona (USA). Three measurements were conducted on powder from the representative pellet to explore potential chemical variations within the feedstock. The results of the chemical analysis for detectable elements are shown in Table 1. Sintered pellets analyzed in this work are Si-lean relative to the anticipated content $(16.4 \%)$ of stoichiometric $\mathrm{U}_{3} \mathrm{Si}_{5}$ by 0.4 weight percent (w/o).

\subsection{Microstructure analysis}

Powder X-ray diffraction (XRD) was performed on a Bruker D8 diffractometer using $\mathrm{CuK} \alpha$ radiation. The instrument was fitted with a graphite monochromator to remove $\mathrm{CuK} \beta$ from the diffracted beam. Measurements were conducted in a Bragg-Brentano geometry from $20-140^{\circ} 2 \theta$ with a step size of $0.01^{\circ}$. Powder specimens were prepared by comminuting ingots using a mortar and pestle. This was done inside an inert atmosphere (He) glovebox to minimize oxygen contamination. The powder was mounted on a Si crystal zero-background plate using a thin layer of vacuum grease, and 
then sealed inside a beryllium container while inside the glovebox . An XRD pattern using the prescribed method collected on a $\mathrm{U}_{3} \mathrm{Si}_{5}$ specimen following powder metallurgy and sintering as described below is shown in Figure 1.

Figure 1 confirms that $\mathrm{U}_{3} \mathrm{Si}_{5}$ is the dominant phase present in the sample. For comparison, the characteristic peaks of other U-Si phases in the vicinity of $\mathrm{U}_{3} \mathrm{Si}_{5}$ are shown, along with the peaks of $\mathrm{UO}_{2}$. A primary peak of $\mathrm{UO}_{2}$ is evident at roughly $28^{\circ}$, and evidence of other peaks at higher angles can be observed. Two peaks at $33^{\circ}$ and $37^{\circ} 2 \theta$ were not identified via the XRD database for any known U-Si or U-O compounds.

Scanning electron microscopy (Inspect, FEI, Hillsboro, Oregon) was also used to further investigate the microstructures of the synthesized materials. Samples of $\mathrm{U}_{3} \mathrm{Si}_{5}$ were cross sectioned, ground, and polished with $0.05 \mu \mathrm{m}$ colloidal silica used as the final step. Although the majority of the polished sample was observed as a single phase, two secondary phases were identified using electron backscatter detection (see Figure 2). The first phase, appearing brighter in the backscatter image indicating higher uranium content, was generally observed as $5-10 \mu \mathrm{m}$ precipitates. These constituted far less than one volume percent. Qualitatively, these precipitates appeared to be somewhat localized with several being collected in a $100 \mu \mathrm{m}$ region but being totally absent from surrounding areas. Energy dispersive spectroscopy (EDS) measurements confirmed that this secondary silicide phase consisted only of $\mathrm{U}$ and $\mathrm{Si}$, and was $\mathrm{U}$-rich compared to the surrounding matrix. It is suspected that the secondary phase is USi based on the accepted phase diagram. However, recent reports [see 7, and references therein] have suggested the existence of $\mathrm{U}_{4} \mathrm{Si}_{5}$, which could also be possible based on the EDS data. Further characterization of the specimen with neutron diffraction or convergent beam electron microscopy would be necessary for decisive assignment.

A second precipitate roughly the same size as the first was also identified in the backscatter SEM images, appearing darker than the matrix. These precipitates were observed less frequently than the U-rich phases. EDS measurement of these precipitates identified the presence of $\mathrm{Si}, \mathrm{U}$, and $\mathrm{O}$. It is assumed that this phase is $\mathrm{UO}_{2}$ given the XRD analysis, and that the Si EDS signal is likely due to the interaction volume of the electron beam penetrating the $5-10 \mu \mathrm{m}$ particles.

\subsection{Differential scanning calorimetry}

Specimens for heat capacity measurements were prepared according to $\S 2.2$ using a $6 \mathrm{~mm}$ punch and die set. Resulting cylindrical pellets weighed 
$0.170 \mathrm{~g}$ and had diameters of $4.58 \mathrm{~mm} \pm 0.01 \mathrm{~mm}$ and thickness of $1.17 \mathrm{~mm}$ $\pm 0.01 \mathrm{~mm}$. Measurements in the DSC used Pt pans with $\mathrm{Al}_{2} \mathrm{O}_{3}$ liners, which prevented reaction of the $\mathrm{U}_{3} \mathrm{Si}_{5}$ phase with the pans and thermocouples. Specimen mass varied by $<0.01 \mathrm{mg}$ between measurements.

The ratio method was utilized to determine the specific heat capacity,* $c_{p}$, of the specimens in a differential scanning calorimeter (Pegasus $404 \mathrm{C}$, Netzsch Instruments, Germany). Measurements were conducted at a heating rate of $20 \mathrm{~K} \cdot \mathrm{min}^{-1}$ to a final temperature of $1243 \mathrm{~K}$. The atmosphere in the DSC was comprised of ultrahigh purity $\operatorname{Ar}(\mathrm{g})$ that flowed through a $\mathrm{Cu}$ getter source (2A-100, Centorr Vacuum Industries, USA), which decreased the $\mathrm{O}_{2}(\mathrm{~g})$ content in the gas stream to $10^{-15} \mathrm{ppm}$. Oxygen content on the gas stream was monitored on the exhaust by an in-stream oxygen sensor (Rapidox 3100 OEM, Cambridge Sensotec, UK) to verify minimal $\mathrm{O}_{2}(\mathrm{~g})$ contamination in the furnace chamber. Thermocouple and sensitivity calibrations were conducted using melt point standards over the entire range of temperatures investigated. Based on the temperature calibrations, the instrument had an error in temperature of $\pm 1 \mathrm{~K}$. Onsets for phase transformations were calculated using a linear intercept method within the software provided by the manufacturer of the equipment [8].

\subsection{Dilatometry}

Thermal expansion specimens were prepared according to $\S 2.2$ using a 6 $\mathrm{mm}$ punch and die set. Mass for a typical thermal expansion specimen was $0.794 \mathrm{~g}$ and had diameters of $4.57 \mathrm{~mm} \pm 0.01 \mathrm{~mm}$ and thicknesses of 5.35 $\mathrm{mm} \pm 0.02 \mathrm{~mm}$.

Measurement of the physical coefficient of thermal expansion, $\alpha_{P}$, was determined using a dilatometer (DIL 402CD, Netzsch Instruments, Germany). A heating rate of $2.5 \mathrm{~K} \cdot \mathrm{min}^{-1}$ was used to a maximum temperature of 1732 K. A baseline correction was applied to the data to correct for the thermal expansion of the $\mathrm{Al}_{2} \mathrm{O}_{3}$ fixturing. The Ar atmosphere was monitored with an oxygen sensor on the outlet. Initial $\chi_{\mathrm{O}_{2}}$ values were $0.1-1 \mathrm{ppm}$, which dropped to $10^{-12} \mathrm{ppm}$ during the measurement and returned to the initial value on cooling. Specimen mass increased by $<0.5 \mathrm{mg}$ between measurements, which is attributed to surface oxidation of the specimen. Approxi-

*Heat capacity values were converted to molar values using a molecular weight of 854.56 $\mathrm{g} \mathrm{mol}^{-1}$. 
mation of the phase transformation temperature was achieved using a linear intercept method similar to the DSC.

\subsection{Thermal diffusivity measurements}

Cylindrical specimens were fabricated according to $\S 2.2$ using a $13 \mathrm{~mm}$ punch and die set. The specimen weights were between $2 \mathrm{~g}$ and $2.7 \mathrm{~g}$ with thickness between $2.19 \mathrm{~mm}$ and $2.95 \mathrm{~mm}$ with a standard error of \pm 0.02 $\mathrm{mm}$. Pellet diameters were $11.4 \mathrm{~mm} \pm 0.1 \mathrm{~mm}$ in size. Diffusivity values were determined by fitting the temperature rise signal with a Cape-Lehman model [9]. Calibration of the thermocouple used to measure the sample temperature was achieved using the Curie temperature of Fe; the minimum in the diffusivity curve produced was located at $1045.2 \mathrm{~K}$, slightly above the accepted value of $1043 \mathrm{~K}$. As such, an uncertainty of $\pm 3 \mathrm{~K}$ is ascribed to the temperatures of each measurement.

The LFA (LFA 427, Netzsch Instruments, Germany) technique was utilized to determine the thermal diffusivity, $D$, of the specimens. In this technique, a laser is pulsed on one side of the specimen, while the heat rise is determined on the opposite side by measuring the infrared blackbody radiation. All surfaces of the sample were coated with a graphite coating (Graphit 33, Kontakt Chemie) to improve absorption of the laser energy on the bottom face and enhance emissivity of the upper face. Diffusivity data was collected from room temperature to $1773 \mathrm{~K}$ in $50 \mathrm{~K}$ increments on cooling. Additional data was collected between $273 \mathrm{~K}$ and $1174 \mathrm{~K}$ on heating and cooling to investigate hysteresis that was observed in the specimens. Reaction temperatures were determined qualitatively using a linear intercept method similar to the DSC. Reactions were observed to be sigmoidal in nature on heating in the diffusivity measurements. Given that two reaction temperature intercepts were determine, the higher reaction temperature value was accepted due to local heating of about $50 \mathrm{~K}$ that is induced by the laser [10] and the value was closer to the temperature observed in the DSC (see also inset of Figure 5). Three laser shots were collected at each temperature step and averaged to produce the diffusivity values reported in this study. $\mathrm{Al}_{2} \mathrm{O}_{3}$ fixturing was used throughout the sample chamber, and gettered argon was again flowed throughout the measurement. Oxygen content of the outlet gas stream was monitored with an oxygen sensor during each measurement and was $<10^{-15} \mathrm{ppm}$ for all measurements. Specimen mass gains were $1-3 \mathrm{mg}$, which is attributed to surface reaction with the graphite coating. 


\section{Results}

Each of the three techniques were used to obtain the datasets as described above. The measured thermal expansion was used to determine the temperature-dependent density based upon room temperature immersion data collected as described in $\S 2.2$. The product of $c_{p}, \rho$, and $D$ results in the thermal conductivity, $\lambda$. The results of all three measurements as well as calculation of the thermal conductivity are discussed below.

\subsection{Coefficient of thermal expansion}

Specimen length change $\left(d L / L_{0}\right)$ and physical coefficient of thermal expansion $\left(\alpha_{p}\right)$ for $\mathrm{U}_{3} \mathrm{Si}_{5}$ are shown in Figure 3 as a function of temperature to $1673 \mathrm{~K}$. The specimen $d L / L_{0}$ increases linearly with temperature over the entire temperature range. Deviations from linearity begin at $726 \mathrm{~K}$ and return to linear behavior at $732 \mathrm{~K}$ (see inset of Figure 3).

\subsection{Heat capacity}

The molar heat capacity of $\mathrm{U}_{3} \mathrm{Si}_{5}$ as determined by the ratio method in the DSC is shown in Figure 4. Values for $c_{p}$ increase linearly over the entire measurement range, with the exception of the endothermic peak with an onset at $723 \mathrm{~K}$. Sato et al. and Miyadai et al. determined the heat capacity of $\mathrm{U}_{3} \mathrm{Si}_{5}$ for $T<40 \mathrm{~K}$ in a magnetometer [11, 12], which is the only $c_{p}$ data in the literature to the authors' knowledge.

The endothermic peak observed in the DSC at $723 \mathrm{~K}$ is an apparent molar heat induced by the enthalpy of an as yet unidentified phase transformation discussed below. Use of DSC to determine heat capacity will result in superposition of such effects, and they must be removed to arrive at the true heat capacity of the material of interest. The linear behavior of the heat capacity measured below and above the peak facilitated a least squares minimization fit to approximate the heat capacity in the region of the phase transformation. This result, plotted in red in Figure 4, was used to determine the thermal conductivity.

\subsection{Thermal diffusivity}

Thermal diffusivity for $\mathrm{U}_{3} \mathrm{Si}_{5}$ as a function of temperature to $1773 \mathrm{~K}$ is shown in Figure 5. Both heating and cooling data are shown, which produce different diffusivity values for $T<1174 \mathrm{~K}$. On heating from room temperature a linear dependence with temperature is observed. Deviations from 
linearity are observed at two different temperatures: $723 \mathrm{~K}$ and $874 \mathrm{~K}$. The positive deviation from linearity at $723 \mathrm{~K}$ is highlighted in the inset of Figure 5 . Above $723 \mathrm{~K}$, the diffusivity continues to increase linearly until a sharp increase is observed for $874<T<1074 \mathrm{~K}$. Above $1074 \mathrm{~K}$, the measured diffusivity values continue to increase linearly until the maximum temperature determined in this study.

Cooling from $1773 \mathrm{~K}$ follows a linear decrease in diffusivity to $723 \mathrm{~K}$, with $R^{2}=0.999$. Values below $723 \mathrm{~K}$ decrease below the linear trend. At $550 \mathrm{~K}$ a discontinuity in the diffusivity is observed, before decreasing to the initial heating values. Cooling to room temperature returned the thermal diffusivity values to the original magnitude. To the authors' knowledge, no previous thermal diffusivity studies have been conducted on $\mathrm{U}_{3} \mathrm{Si}_{5}$.

\subsection{Thermal conductivity}

The calculated thermal conductivity of $\mathrm{U}_{3} \mathrm{Si}_{5}$ is shown in Figure 6 as a function of temperature to $1773 \mathrm{~K}$. A propagation of errors analysis was conducted based on the errors introduced from measuring $\alpha_{p}, c_{p}$, and $D$ from the DIL, DSC, and LFA techniques, respectively. The result of the error analysis calculates the error in thermal conductivity values at $5 \%$, consistent with that expected of the LFA technique. Thermal diffusivity data on heating was also neglected in this analysis. 


\section{Discussion}

\subsection{Solid state reaction at $723 \mathrm{~K}$}

The existence of a phase transformation is observed at $723 \mathrm{~K}$ in each of the thermophysical property measurements. The closest reaction on the most recent phase diagram [5] is the peritectoid reaction at $723 \mathrm{~K}$, reproduced in Figure 7. This reaction was first postulated by Brown and Norreys [13], who concluded that $\mathrm{USi}_{2}$ decomposes to $\mathrm{USi}_{3}$ and $\mathrm{USi}_{1.88}$ and later confirmed to be exothermic in nature. Observation of a reaction at this same exact temperature in nominally $\mathrm{U}_{3} \mathrm{Si}_{5}$ samples would initially suggest severe offstoichiometry or incomplete arc melting, as slightly $\mathrm{Si}$-rich $\mathrm{U}_{3} \mathrm{Si}_{5}$ would be expected to contain small amounts of $\mathrm{USi}_{1.88}$ rather than $\mathrm{USi}_{2}$. However, XRD, elemental analysis by ICP-OES, and SEM results described above confirm that the samples synthesized here are of the composition and room temperature structure attributed to $\mathrm{U}_{3} \mathrm{Si}_{5}$, and are devoid of any substantial fraction secondary U-Si phase. Furthermore, the DSC signal is endothermic suggesting a different reaction mechanism applies.

Therefore, the results of this study reproduce the reported peritectoid reaction temperature, but the composition and energetics are inconsistent with the literature. A more detailed investigation of previous studies reveals conflicting results regarding phase stability in the U-Si system near the $\mathrm{U}_{3} \mathrm{Si}_{5}$ composition. Results of Remschnig et al. suggest that $\mathrm{U}_{3} \mathrm{Si}_{5}$ is actually a highly defective $\mathrm{AlB}_{2}$-type structure, which exists as two isostructurally similar phases that form superstructures along the c-axis of the unit cell when annealed above $973 \mathrm{~K}$ [14]. Vaugoyeau et al. also investigated a number of U-Si melt compositions near $\mathrm{U}_{3} \mathrm{Si}_{5}$ and observed that $\mathrm{U}_{3} \mathrm{Si}_{5}$ and $\mathrm{USi}_{1.88}$ each have a range of allowed stoichiometries, and are difficult to distinguish by XRD [15].

Further uncertainty is encountered when the sources of $\mathrm{USi}_{2}$ structural data are consulted. Sasa et al. studied $\mathrm{USi}_{2}$ in detail, and reports that the phase is metastable and will degrade exothermically at $623 \mathrm{~K} \mathrm{[16].} \mathrm{Stoi-}$ chiometric $\mathrm{USi}_{2}$ characterized in the work of Sasa et al. was synthesized by leaching excess $\mathrm{U}$ from $\mathrm{USi}_{1.88}$ in nitric acid. Other techniques that report data for $\mathrm{USi}_{2}$ utilize a bismuth amalgam technique $[17,13]$. To the authors' knowledge, no previous investigators have synthesized $\mathrm{USi}_{2}$ using a steady state technique.

The data of this study supports the conclusion that the U-Si phase diagram as shown in Figure 7, while accurate at room temperature in the vicinity 
of $\mathrm{U}_{3} \mathrm{Si}_{5}$, contains inaccuracies at elevated temperatures. The results of this study do not provide any conclusions with respect to phase transformations at elevated temperatures, but it can be stated that a U-Si compound synthesized at the target composition of $\mathrm{U}_{3} \mathrm{Si}_{5}$ undergoes an endothermic phase transformation at $723 \mathrm{~K}$. The reaction is shown to be reversible upon cooling; hysteresis of the thermal conductivity and possible implications of this response to the use of $\mathrm{U}_{3} \mathrm{Si}_{5}$ as reactor fuel are discussed below. Diffraction studies at elevated temperatures are needed to elucidate the complete structural behavior of this compound, but are beyond the scope of this current study.

\subsection{Density dependence of $U_{3} S i_{5}$ with temperature}

The physical coefficient of thermal expansion is solved by taking the instantaneous derivative of $d L / L_{0}$ with temperature, which is shown in Figure 3. An average value for $\alpha_{p}$ is calculated from the data at $10.7 \times 10^{-6}$ $\mathrm{K}^{-1} \pm 0.5 \times 10^{-6} \mathrm{~K}^{-1}$ for $300 \mathrm{~K}<T<1250 \mathrm{~K}$, which is plotted as the red-line. Above $1250 \mathrm{~K}, d L / L_{0}$ and corresponding $\alpha_{p}$ values deviate slightly from linearity, which increases the error in assuming a constant $\alpha_{p}$ to $\pm 2 \times 10^{-6} \mathrm{~K}^{-1}$. Using standard error analysis techniques, the error in assuming a constant $\alpha_{p}$ generates an error in density of $<1 \%$ over the entire temperature range.

\subsection{Fundamental contributions to the molar heat capacity of $U_{3} S_{5}$}

The molar heat capacity of metals at constant pressure is predominantly composed of harmonic lattice, anharmonic lattice, dilational, and electronic terms. The dilational term is calculated using the well known relation $c_{d}=$ $\alpha_{p}^{2} V k_{T}^{-1} T$, where $V$ and $k_{T}$ are the molar volume and isothermal compressibility, respectively. Compressibility values for $\mathrm{U}_{3} \mathrm{Si}_{5}$ were not found during the literature review. Values for the bulk modulus of $\mathrm{USi}_{2}$ and $\mathrm{USi}$ are reported by Yagoubi et al. to be $140 \mathrm{GPa}$ and $111 \mathrm{GPa}$, respectively [18]. Assuming that the bulk modulus for $\mathrm{U}_{3} \mathrm{Si}_{5}$ is similar to $\mathrm{USi}_{2}$ provides an estimate to the dilatational heat capacity term. Using this value for $k_{T}$ and $\alpha_{p}$ value provided in the previous section, the dilational contribution to heat capacity is calculated, which contributes less than $0.1 \%$ over the entire temperature range analyzed in this study.

Contribution to heat capacity by electronic, $c_{e}$, and anharmonic lattice, $c_{a}$, terms follow a linear dependence with temperature. Due to the complex nature of modeling the anharmonic term, it is common for this term to be summed with the electronic term as $(b+\gamma) T$, where $b$ and $\gamma$ are the 
anharmonic and electronic constants, respectively. By expanding the Debye model, one can solve for the linear component using [see 19, and references therein]:

$$
\left(c_{v}+c_{e}-3 N R\right) T^{-1}=-\frac{3 N R}{20} \frac{\Theta_{D}^{2}}{T^{3}}+(b+\gamma)
$$

where $N, R$ and $\Theta_{D}$ are the number of atoms in the unit cell, gas constant and the Debye temperature, respectively. A least squares fit of Equation (1) is shown in the inset of Figure 4. The value of $(b+\gamma)$ for $\mathrm{U}_{3} \mathrm{Si}_{5}$ from this study is $30.5 \mathrm{~mJ} \cdot \mathrm{mol}^{-1} \cdot \mathrm{K}^{-2}\left(\mathrm{U}_{3} \mathrm{Si}_{5}\right.$ basis), which is within $0.5 \%$ with the value calculated from low temperature heat capacity data [11, 12]. A value of $183 \mathrm{~K}$ is calculated for $\Theta_{D}$ based on the slope of the aforementioned fit. A comparison of $\Theta_{D}$ with the literature is not available to the author's knowledge. Subtracting the electronic and dilational contribution from the heat capacity data calculates $c_{v}$, shown as the $(\diamond)$ symbols in Figure 4 . For $T<1000 \mathrm{~K}, c_{v}$ is within $1 \%$ of the Dulong-Petit value $\left(199.5 \mathrm{~J} \cdot \mathrm{mol}^{-1} \cdot \mathrm{K}^{-1}\right)$, which is expected given the calculated Debye temperature for this system. Above $1000 \mathrm{~K}, c_{v}$ is at most $4 \%$ lower than the Dulong-Petit value. For the purposes of calculating the thermal conductivity, a linear regression analysis was assumed over the entire temperature range, represented by the red line in Figure 4.

\subsection{Thermal diffusivity and thermal conductivity dependence with tempera- ture for $\mathrm{U}_{3} \mathrm{Si}_{5}$}

The material characterized in this study can be declared of acceptably high purity based on the XRD and SEM analyses. Although a small quantity of $\mathrm{UO}_{2}$ was observed in the microstructure, which would not affect the thermal diffusivity data. The error introduced to the thermal conductivity of $\mathrm{U}_{3} \mathrm{Si}_{5}$ from second phases limited to at most a few percent each will be less than the error of the calculation (5\%), and as such can be considered to be captured by this term.

Thermal transport in metallic materials can be simplified to transport of thermal energy by phonons and electrons in this temperature range. In general, the phonon term in metallic conductors is only relevant at lower temperatures $(T<100 \mathrm{~K})$ because the thermal conductivity is inversely dependent on temperature, while the electronic term is linearly dependent. Over the measured temperature range, a linear temperature dependence is observed suggesting $\mathrm{U}_{3} \mathrm{Si}_{5}$ is dominated by the electronic conduction term. 
It is known that the electronic component of the thermal conductivity in metals decreases upon the formation of defects in the solid state [20]. Nonlinear increases in thermal diffusivity for $874<T<1074 \mathrm{~K}$ on heating (see Figure 5) are consistent with the removal of the defective Pmmm structure in this material on heating $\mathrm{U}_{3} \mathrm{Si}_{5}$ as reported by Remchnig et al. [14]. Interestingly, the thermal diffusivity data suggests that some degree of hysteresis exists in this phase field from the kinetics of defect removal in the system as evidenced by differential heating and cooling. As such, thermal conductivity data represented by the (- - ) in Figure 6 should be taken with caution and depends on the specimen history. It is suspected that the defective structure does not form on cooling until well below $723 \mathrm{~K}$, although more detailed diffraction work as a function of temperature is necessary to evaluate this hypothesis. Furthermore, the sharp transition observed at $T_{t r}=550 \mathrm{~K}$ is not understood given the analysis techniques utilized in this study. Further diffraction work is again required to elucidate the fundamental mechanisms involved in the transition.

The thermal conductivity of $\mathrm{UO}_{2}$, which is dominated by phonon transport in the temperature range of this study, is shown as a function of temperature in Figure 6 [21]. At room temperature, the thermal conductivity of $\mathrm{U}_{3} \mathrm{Si}_{5}$ is $\sim 60 \%$ lower in value than $\mathrm{UO}_{2}$. On heating, the thermal conductivity of $\mathrm{U}_{3} \mathrm{Si}_{5}$ overtakes the $\mathrm{UO}_{2}$ values at $574 \mathrm{~K}$ and continues to increase with temperature. For comparison, data for UN from Hayes et al. is also included [22]. Thermal conduction in UN has a similar metallic behavior to $\mathrm{U}_{3} \mathrm{Si}_{5}$ although the magnitude for $\mathrm{UN}$ is $\sim 60 \%$ larger over the entire temperature range conducted in this study.

It is expected that $\mathrm{U}_{3} \mathrm{Si}_{5}$ is metastable on heating above or cooling below the reaction temperature at $723 \mathrm{~K}$, which impacts the thermal transport in this system. For a reactor application, it is reasonable that portions of $\mathrm{U}_{3} \mathrm{Si}_{5}$ fuel elements would operate for extended periods of time within the temperature region where the phase transformation is observed. At a minimum, the hypothesized phase transformation has been demonstrated in this work to perturb the thermal conductivity of $\mathrm{U}_{3} \mathrm{Si}_{5}$ by over $30 \%$. Such an uncertainty in the thermal conductivity of a reactor fuel at service temperatures would require conservatisms detrimental to the demands of commercial units. Unknowns regarding the kinetics of defect recovery in this system were not investigated in this study, but are relevant to understanding $\mathrm{U}_{3} \mathrm{Si}_{5}$ as a fuel element. Clearly the performance of $\mathrm{U}_{3} \mathrm{Si}_{5}$ under irradiation testing is paramount to validating this fuel in a reactor setting. However, the details of 
414 this phase transformation, as well as the impact of irradiation on the stability 415 of highly-defective structures that are reported to populate this portion of ${ }_{416}$ the U-Si binary system, are first essential even before in-pile testing if $\mathrm{U}_{3} \mathrm{Si}_{5}$ 417 is to be considered for nuclear reactor applications. 


\section{Conclusions}

The coefficient of thermal expansion, molar heat capacity, thermal diffusivity, and thermal conductivity of $\mathrm{U}_{3} \mathrm{Si}_{5}$ were determined as a function of temperature to $1773 \mathrm{~K}$. A constant coefficient of thermal expansion was determined over the entire temperature range of $10.7 \times 10^{-6} \mathrm{~K}^{-1}$. Electronic contributions to the molar heat capacity measured in this study were consistent with values presented in the literature and the Dulong-Petit law is observed once the electronic component is neglected. Metallic thermal conduction is observed in $\mathrm{U}_{3} \mathrm{Si}_{5}$ which increases linearly with temperature. An endothermic phase transformation was observed at $723 \mathrm{~K}$ in all measurements executed here. Although this temperature is consistent with the $\mathrm{USi}_{2}$ peritectoid reaction located further into the Si-rich portion of the binary, neither XRD nor SEM revealed its existence in samples synthesized here.

\section{Acknowledgements}

The support of the U. S. Department of Energy, Office of Nuclear Energy Fuel Cycle Research and Development program is gratefully acknowledged. This work was performed at Los Alamos National Laboratory under the auspices of the U.S. Department of Energy. 


\section{References}

[1] Y.-S. Kim, Uranium intermetallic fuels (U-Al, U-Si, U-Mo), in: R. Konings (Ed.), Comprehensive Nuclear Materials, Vol. 3, Elsevier Ltd, 2012, pp. 391-420.

[2] N. R. Brown, A. Aronson, M. Todosow, R. Brito, K. J. McClellan, Neutronic performance of uranium nitride composite fuels in a PWR, Nuclear Engineering and Design 275 (2014) 393 - 407.

[3] A. Nelson, J. White, D. Byler, J. Dunwoody, J. Valdez, K. McClellan, Overview of properties and performance of uranium-silicide compounds for light water reactor applications, Transactions of the American Nuclear Society 110 (2014) 987-989.

[4] J. White, A. Nelson, D. Byler, J. Valdez, K. McClellan, Thermophysical properties of $\mathrm{U}_{3} \mathrm{Si}$ to $1150 \mathrm{~K}$, Journal of Nuclear Materials 452 (1-3) (2014) $304-310$.

[5] H. Okamoto, Si-U (Silicon-Uranium), in: T. Massalski (Ed.), Binary Alloy Phase Diagrams, Second Edition, Vol. 3, ASM International, 1990, pp. 3374-3375.

[6] Standard test method for density of compacted or sintered powder metallurgy (PM) products using archimedes' principle, ASTM International, West Conshohocken, PA, B962-08 (2008).

[7] A. Berche, C. Rado, O. Rapaud, C. Guneau, J. Rogez, Thermodynamic study of the U-Si system, Journal of Nuclear Materials 389 (1) (2009) $101-107$.

[8] Proteus Analysis Software, version 5.2.0, Netzsch-Gerätebau, GmbH, Selb, Germany, 2010.

[9] J. A. Cape, G. W. Lehman, Temperature and finite pulsetime effects in the flash method for measuring thermal diffusivity, Journal of Applied Physics 34 (7) (1963) 1909-1913.

[10] W. J. Parker, R. J. Jenkins, C. P. Butler, G. L. Abbott, Flash method of determining thermal diffusivity, heat capacity, and thermal conductivity, Journal of Applied Physics 32 (9) (1961) 1679-1684. 
[11] N. Sato, M. Kagawa, K. Tanaka, N. Takeda, T. Satoh, T. Komatsubara, Magnetic properties of a mass-enhanced ferromagnet $\mathrm{U}_{2} \mathrm{PtSi}_{3}$, Journal of Magnetism and Magnetic Materials 108 (1-3) (1992) 115 - 116.

[12] T. Miyadai, H. Mori, T. Oguchi, Y. Tazuke, H. Amitsuka, T. Kuwai, Y. Miyako, Magnetic and electrical properties of the U-Si system (part ii), Journal of Magnetism and Magnetic Materials 104-107 (1992) 47 48 .

[13] A. Brown, J. J. Norreys, Uranium disilicide, Nature 191 (4783) (1961) $61-62$.

[14] K. Remschnig, T. L. Bihan, H. Nöel, P. Rogl, Structural chemistry and magnetic behavior of binary uranium silicides, Journal of Solid State Chemistry 97 (2) (1992) 391 - 399.

[15] H. Vaugoyeau, L. Lombard, J. Morlevat, Contribution a l'etude du diagramme d'equilibre uranium silicium, Journal of Nuclear Materials 39 (3) (1971) 323 - 329.

[16] Y. Sasa, M. Uda, Structure of stoichiometric USi ${ }_{2}$, Journal of Solid State Chemistry 18 (1) (1976) $63-68$.

[17] A. Brown, J. J. Norreys, Beta-polymorphs of uranium and thorium disilicides, Nature 183 (4662) (1959) 673 - 673.

[18] S. Yagoubi, S. Heathman, A. Svane, G. Vaitheeswaran, P. Heines, J.-C. Griveau, T. L. Bihan, M. Idiri, F. Wastin, R. Caciuffo, High pressure studies on uranium and thorium silicide compounds: Experiment and theory, Journal of Alloys and Compounds 546 (2013) $63-71$.

[19] J. Nakamura, M. K. Yochi Takahashi, S. Izumi, Heat capacity of metallic uranium and thorium from 80 to $1000 \mathrm{~K}$, Journal of Nuclear Materials 88 (1) (1980) $64-72$.

[20] K. Mendelssohn, Electrical and thermal conductivity of metals, Canadian Journal of Physics 34 (12A) (1956) 1315-1327.

[21] J. White, A. Nelson, Thermal conductivity of $\mathrm{UO}_{2+x}$ and $\mathrm{U}_{4} \mathrm{O}_{9-y}$, Journal of Nuclear Materials 443 (1-3) (2013) $342-350$. 
[22] S. Hayes, J. Thomas, K. Peddicord, Material property correlations for uranium mononitride: IV. Thermodynamic properties, Journal of Nuclear Materials 171 (2-3) (1990) $300-318$.

[23] H. Weitzel, C. Keller, Neutronenbeugungsuntersuchungen an $\left(\mathrm{SE}_{0.5} \mathrm{U}_{0.5}\right) \mathrm{O}_{2} \quad(\mathrm{SE}=\mathrm{Y}, \mathrm{La}, \mathrm{Nd}$, Ho, und Lu), Journal of Solid State Chemistry 13 (1-2) (1975) 136 - 141.

[24] W. H. Zachariasen, Crystal chemical studies of the $5 f$-series of elements. VIII. Crystal structure studies of uranium silicides and of $\mathrm{CeSi}_{2}, \mathrm{NpSi}_{2}$, and $\mathrm{PuSi}_{2}$, Acta Crystallographica 2 (2) (1949) 94-99. 
Table 1: Chemical analysis conducted on as-sintered $\mathrm{U}_{3} \mathrm{Si}_{5}$ pellets. Elements included in the analysis, but below the detection limit are included as follows: $\mathrm{Al}, \mathrm{Sb}, \mathrm{Ba}, \mathrm{Be}, \mathrm{Cd}$, $\mathrm{Ca}, \mathrm{Cr}, \mathrm{Fe}, \mathrm{Pb}, \mathrm{Li}, \mathrm{Mg}, \mathrm{Mo}, \mathrm{Ni}, \mathrm{P}, \mathrm{K}, \mathrm{Se}, \mathrm{Na}, \mathrm{Sr}, \mathrm{Sn}, \mathrm{Ti}, \mathrm{Zn}, \mathrm{Zr}, \mathrm{B}, \mathrm{Gd}, \mathrm{Nb}, \mathrm{Ta}$, and W.

$\begin{array}{lllllllllll}\text { Elements } & \mathrm{Si} & \mathrm{C} & \mathrm{H} & \mathrm{N} & \mathrm{Cu} & \mathrm{V} & \mathrm{As} & \mathrm{Co} & \mathrm{Mn} & \mathrm{Hf}\end{array}$

$\begin{array}{lllllllllll}\text { Contents (w/o) } & 16.00 & 0.23 & 0.27 & 0.16 & 0.008 & 0.005 & 0.03 & 0.005 & 0.01 & 0.02\end{array}$




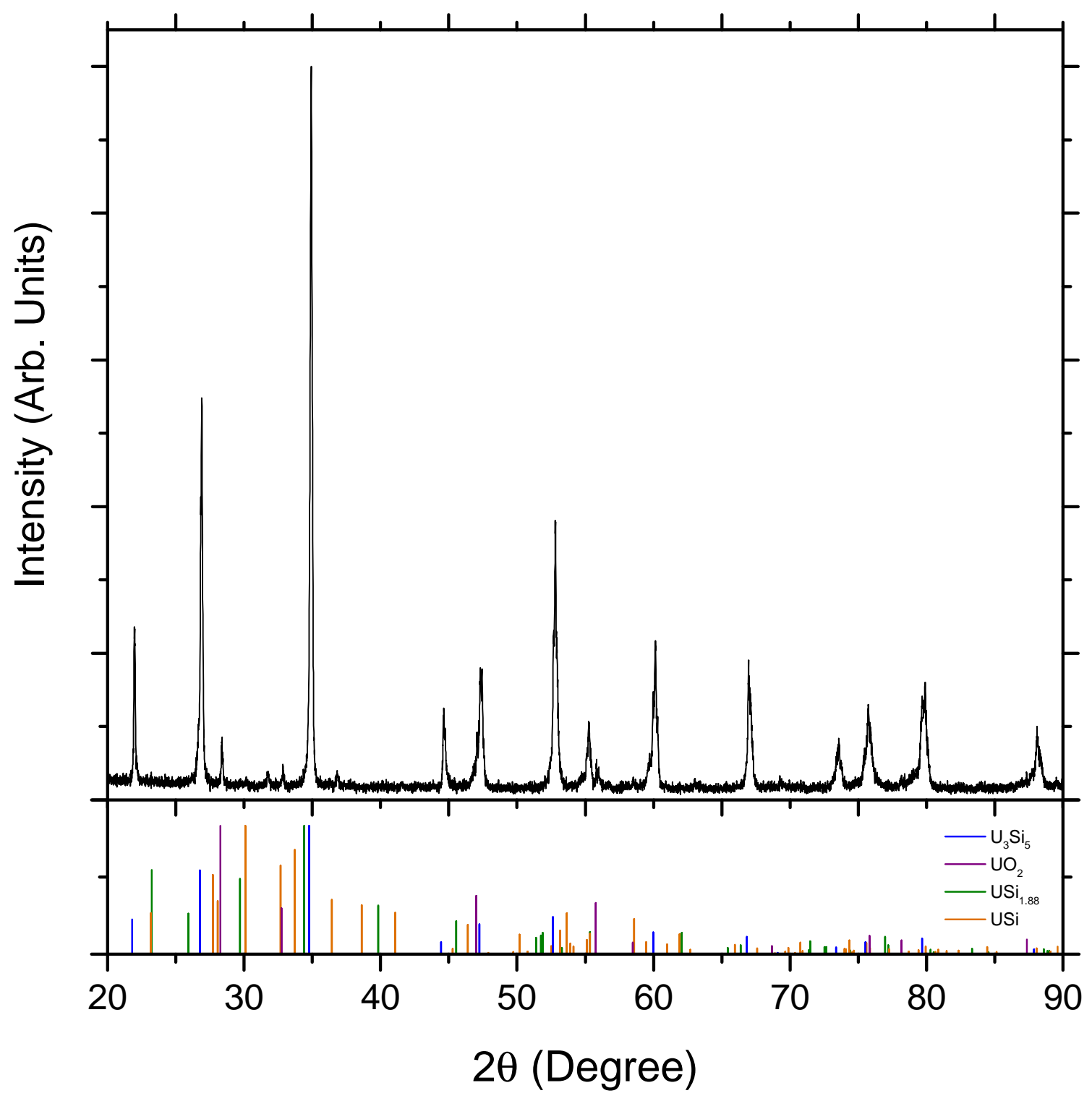

Figure 1: XRD pattern collected on a sintered $\mathrm{U}_{3} \mathrm{Si}_{5}$ specimen that was crushed and sealed within a Be sample holder. Reference files are from: 04-003-3432 $\left(\mathrm{U}_{3} \mathrm{Si}_{5}\right)$ [11], 04-008-2456 $\left(\mathrm{UO}_{2}\right)$ [23], 04-003-0522 (USi) [24], and 04-003-3437 (USi $\left.{ }_{1.88}\right)$ [12]. 


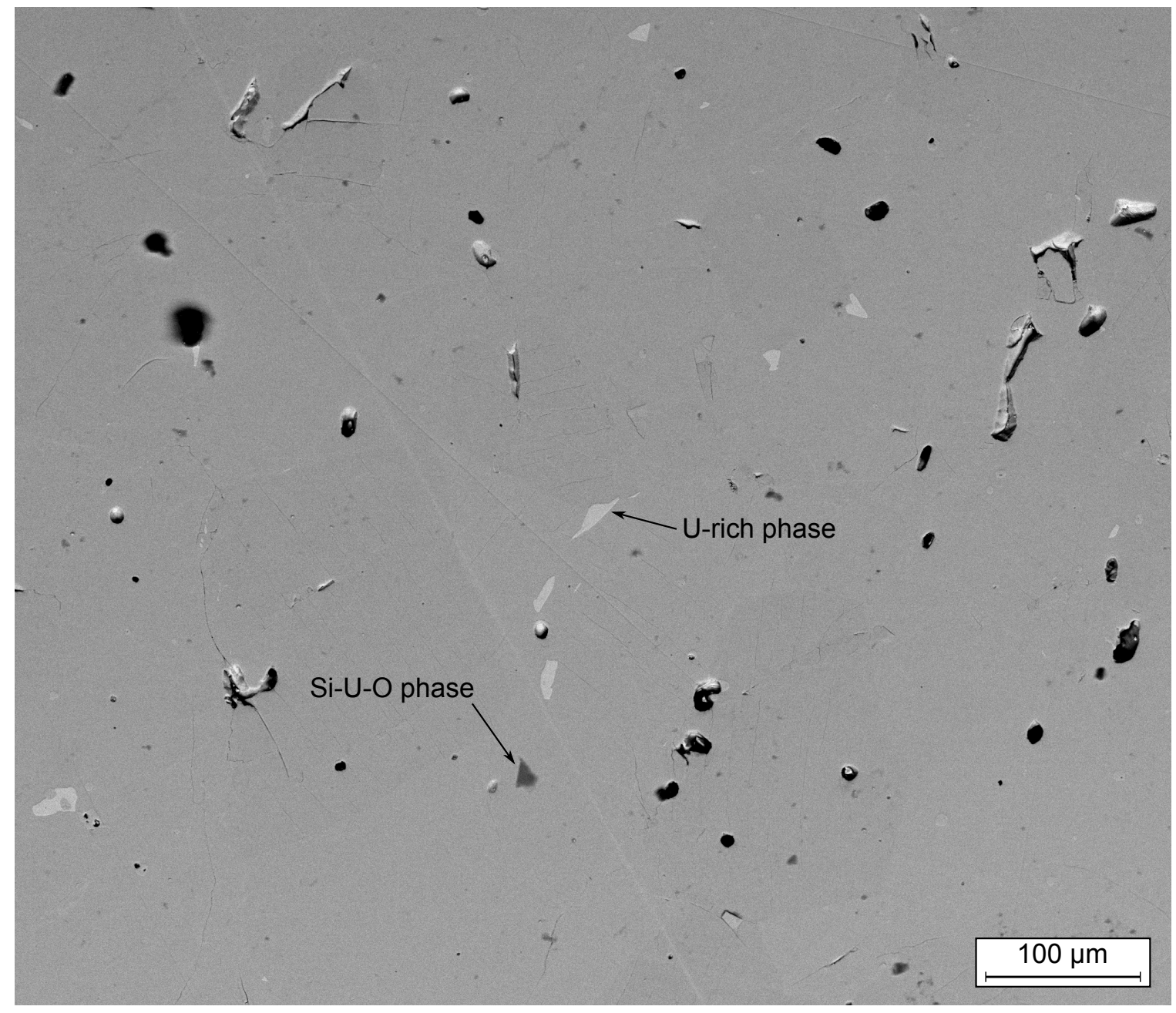

Figure 2: Scanning electron microscope of the $\mathrm{U}_{3} \mathrm{Si}_{5}$ microstructure collected with a backscatter electron detector. 


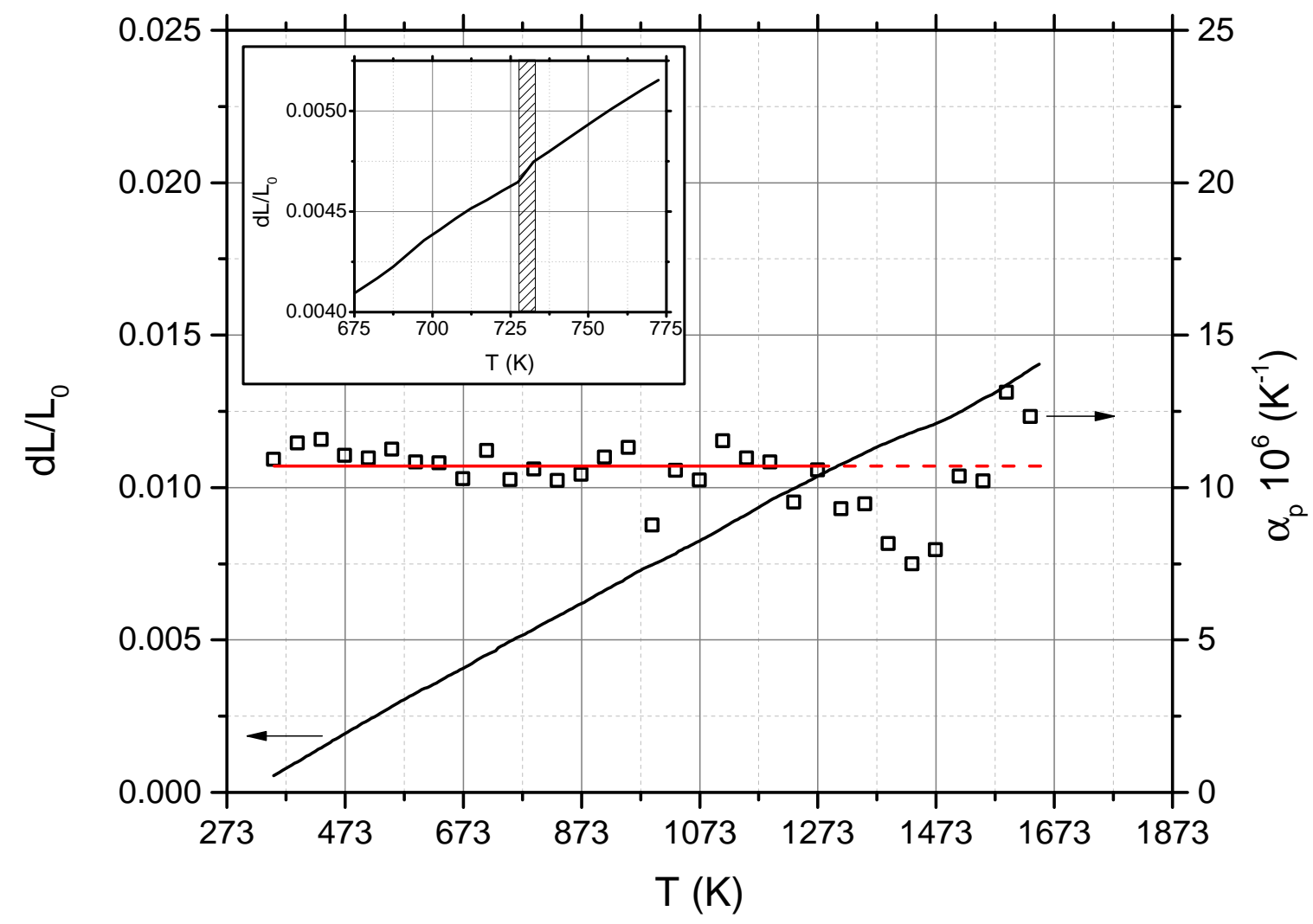

Figure 3: Specimen length change normalized to original specimen length and physical coefficient of thermal expansion as a function of temperature for $\mathrm{U}_{3} \mathrm{Si}_{5}$. The calculated average $\alpha_{p}$ value is shown as the red line. An inset for $d L / L_{0}$ centered at the phase transformation temperature, which is highlighted by the meshed rectangle. 


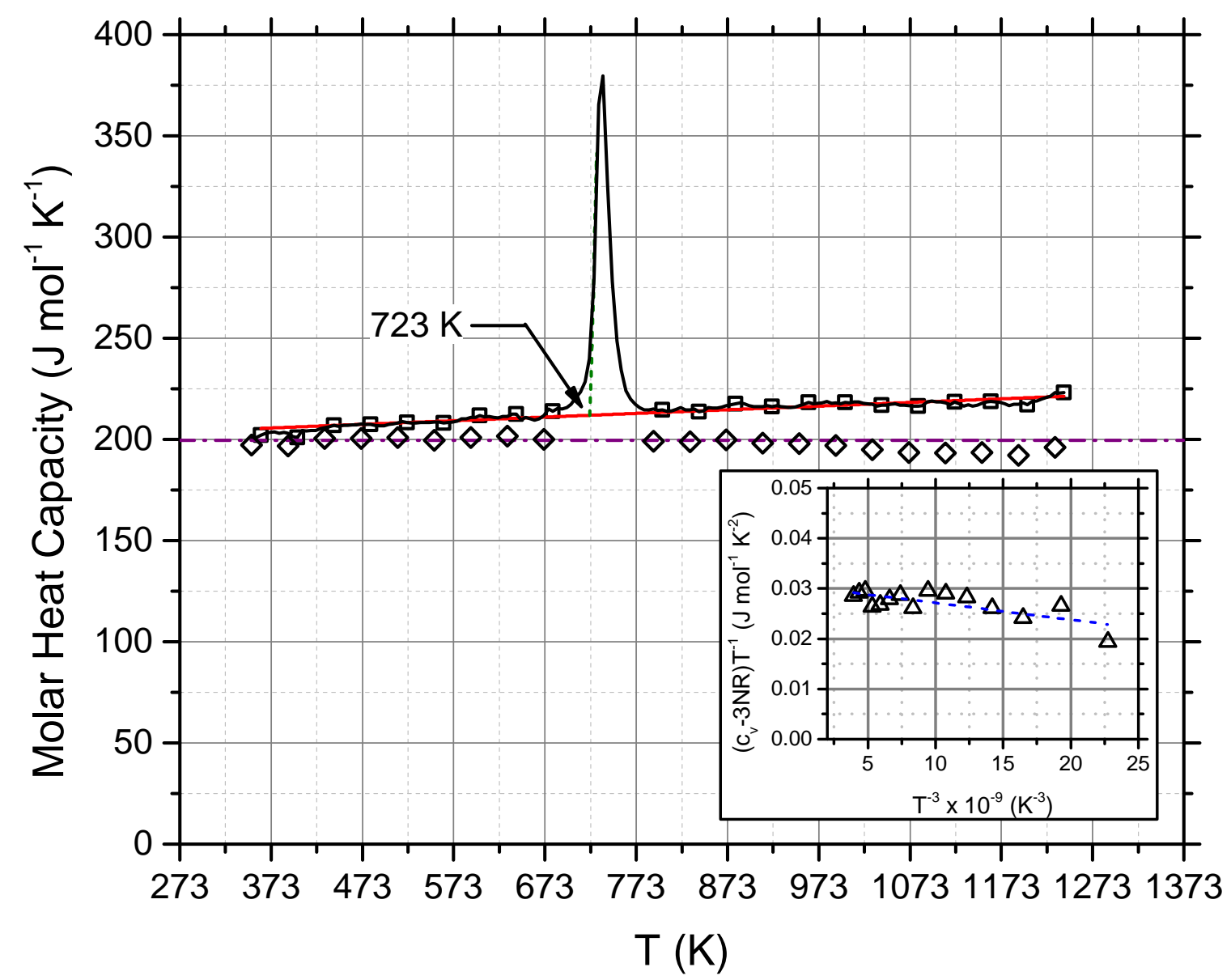

Figure 4: Molar heat capacity of $\mathrm{U}_{3} \mathrm{Si}_{5}$ collected from $353 \mathrm{~K}$ to $1423 \mathrm{~K}$ ( $\square$ ), with calculated $c_{v}(\diamond)$ and Dulong-Petit value $(-\cdot-)$. Least squares minimization fit of the data ( - , which was used to calculate the thermal conductivity. The inset includes a $\left(c_{v}-3 N R\right) T^{-1}$ against $T^{-3}$ plot with a linear fit according to Equation (1). 


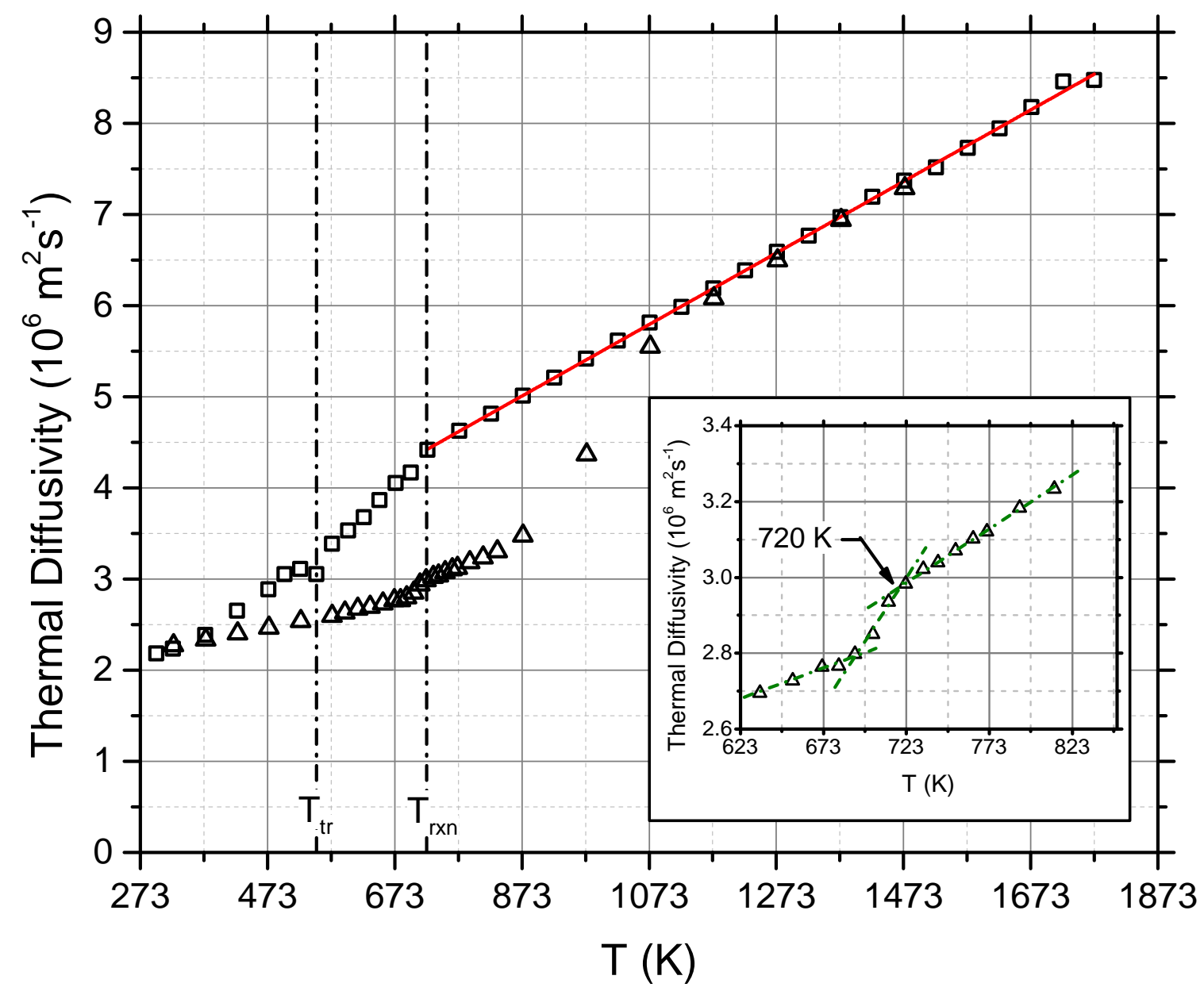

Figure 5: Thermal Diffusivity data collected on heating $(\triangle)$ and cooling $(\square)$ for $\mathrm{U}_{3} \mathrm{Si}_{5}$. The inse includes a meshed tolines are added to the inset, which highlight the heating data near the phase transformation temperature at $723 \mathrm{~K}$. Specific attention is drawn to the qualitatively assessment of the phase transformation temperature in the inset, which is further described in the text. 


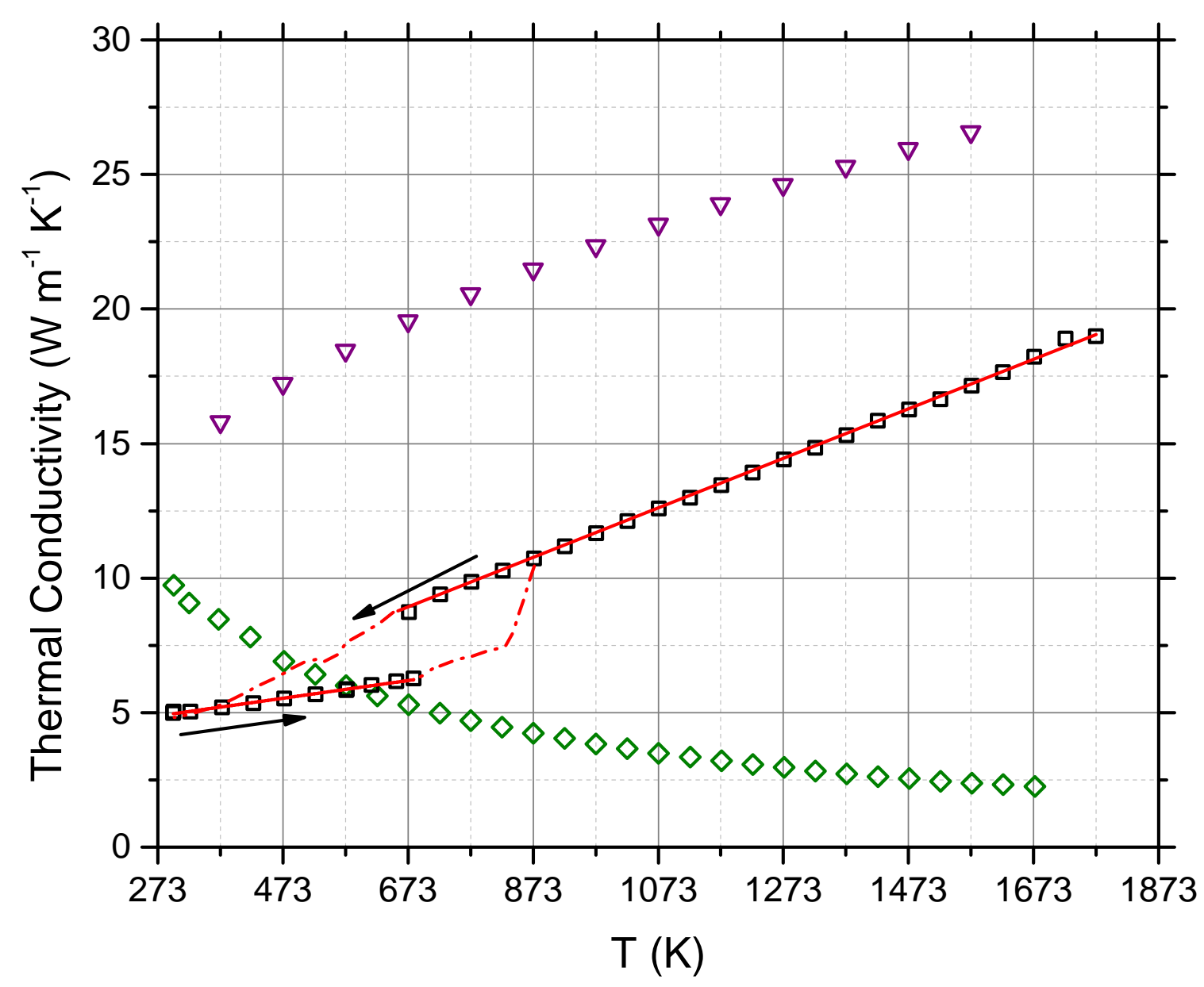

Figure 6: Thermal conductivity data as a function of temperature for $\mathrm{U}_{3} \mathrm{Si}_{5}(\square)$ with a least squares minimization fit of the data $(-)$. Arrows indicate differences in heating and cooling values for $\mathrm{U}_{3} \mathrm{Si}_{5}$. Reference data for $\mathrm{UO}_{2}(\diamond,[21])$ and $\mathrm{UN}(\nabla,[22])$ are included for comparison. 


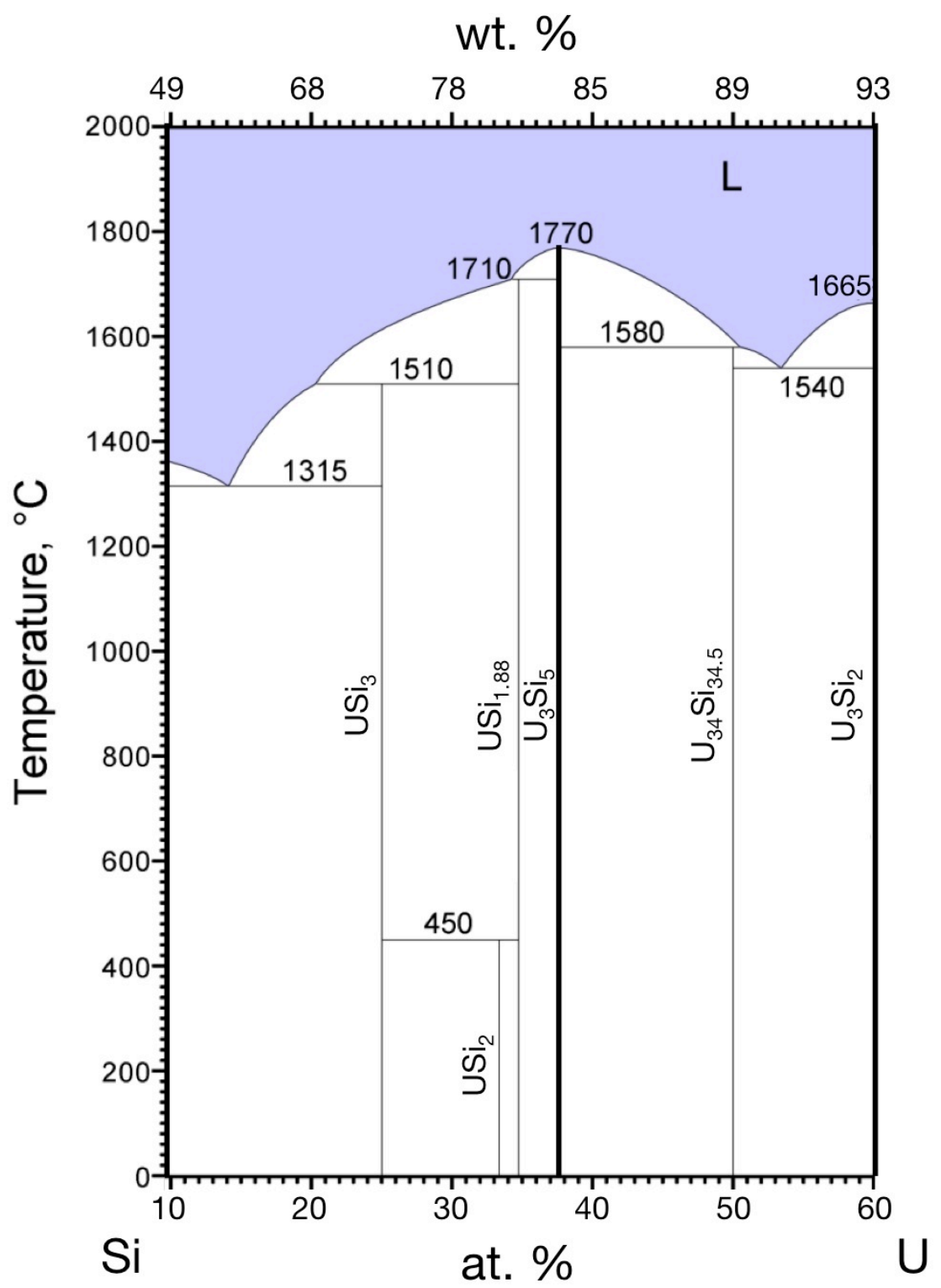

Figure 7: U-Si partial phase diagram based on the full binary as reported by Okamoto [5]. 